\title{
Notions of Independence: Examples and Properties
}

\author{
Bruno Bandeira ${ }^{1}$, Márcia R. Cerioli ${ }^{2}$, Petrucio Viana ${ }^{3}$ \\ ${ }^{1}$ Programa de Engenharia de Sistemas e Computação - COPPE \\ Universidade Federal do Rio de Janeiro (UFRJ) - Rio de Janeiro - RJ - Brazil \\ Bolsista CAPES \\ ${ }^{2}$ Instituto de Matemática e \\ Programa de Engenharia de Sistemas e Computação - COPPE \\ Universidade Federal do Rio de Janeiro (UFRJ) - Rio de Janeiro - RJ - Brazil \\ ${ }^{3}$ Instituto de Matemática e Estatística \\ Universidade Federal Fluminense (UFF) - Niterói - RJ - Brazil \\ brunobmecos.ufrj.br, marcia@cos.ufrj.br, petrucio_vianadid.uff.br
}

\begin{abstract}
We analyze the notions of independence of a set of postulates, proposed by G. Peano, E. H. Moore, A. Church and F. Harary, together with a presumably new notion inspired by $H$. M. Sheffer. For each of these notions, we present a proper definition and give illustrative examples. We give a complete picture of the implications between these notions of independence. Then, we make some additional observations that shed some light on these notions and some concepts related to them.
\end{abstract}

\section{Introduction}

In this paper, we review and analyse some notions of independence of sets of postulates which appear in the bibliography on mathematical logic. More specifically, we review what it means for a set of postulates to be independent [Peano 1889], completely independent [Moore 1910], irredundant [Church 1925], prime (inspired by [Sheffer 1916]), strongly independent [Harary 1961], and absolutely independent [Harary 1961]. In addition, we investigate the relationships between these notions, determining, from a logical point of view, which are weaker, equivalent or stronger than the others. Then, within an original perspective, we give a closer look at the notion of irredundance, showing that it is equivalent to the existence and uniqueness of a minimal subset of the postulates that imply a given consequence. Finally, we show that, under some mild conditions, strong independence is implied by irredundance. We explain the concepts in detail, but we leave out some of the proofs to make the paper fit the required length. Nevertheless, all proofs will be published in full detail in an extensive paper that is currently in preparation.

\section{Preliminaries}

In the following sections, we deal with notions of independence that are applicable to sets of sentences belonging to some well-known formalisms-such as Propositional Logic, First Order Logic, and Second Order Logic. We assume that a formalism must have the following characteristics:

(1) There is a distinguished non-empty set $\mathcal{L}$ whose elements are called sentences, which are generically denoted by $\varphi, \psi$, and $\theta$. 
(2) There is a distinguished non-empty class $\mathcal{S}$ whose elements are called structures, which are generically denoted by $\mathfrak{M}, \mathfrak{N}$, and $\mathfrak{O}$.

(3) There is a function Mod which associates to each $\varphi \in \mathcal{L}$ a subclass $\operatorname{Mod}(\varphi) \subseteq \mathcal{S}$, referred to as the class of models of $\varphi$. Hence, given $\varphi$ and $\mathfrak{M}$, we say that $\mathfrak{M}$ is a model of $\varphi$, or that $\mathfrak{M}$ satisfies $\varphi$, when $\mathfrak{M} \in \operatorname{Mod}(\varphi)$. Besides, given $\Sigma \subseteq \mathcal{L}$, we define $\operatorname{Mod}(\Sigma)=\bigcap\{\operatorname{Mod}(\varphi): \varphi \in \Sigma\}$. Finally, we say that $\varphi$ is valid, and write $\models \varphi$, when $\operatorname{Mod}(\varphi)=\mathcal{S}$.

We assume, for all formalisms treated here, that the set of sentences is endowed withat least - the three distinguished classical operations of negation, conjunction, and disjunction. Whence, all formalisms which concern us will contain, for all sentences $\varphi$ and $\psi$, also their negations $\neg \varphi$, conjunctions $\varphi \wedge \psi$, and disjunctions $\varphi \vee \psi$ as sentences. Accordingly, we take for granted the following semantical properties: for all sentences $\varphi$ and $\psi, \operatorname{Mod}(\neg \varphi)=\mathcal{S} \backslash \operatorname{Mod}(\varphi) \operatorname{Mod}(\varphi \wedge \psi)=\operatorname{Mod}(\varphi) \cap \operatorname{Mod}(\psi)$ and $\operatorname{Mod}(\varphi \vee \psi)=\operatorname{Mod}(\varphi) \cup \operatorname{Mod}(\psi)$.

\section{Definitions of Independence}

In this section, we present the notions of independence we are dealing with. For each notion, we present a proper definition and give illustrative examples.

\subsection{Independence (Peano, 1891)}

The first notion of independence is due to G. Peano. Usually, when we talk about the independence of axioms or postulates, it is this notion that we are referring to.

Definition 1 [Peano 1891] $\Sigma$ is independent if $\operatorname{Mod}[(\Sigma \backslash\{\varphi\}) \cup\{\neg \varphi\}] \neq \emptyset$, for every $\varphi \in \Sigma$.

Below are two examples of independent sets of sentences. Neither of them satisfy the other forms of independence defined in Sections $3.2-3.5$.

Example 1 (a) In Propositional Logic, the set $\{p, q,(p \vee q) \wedge r\}$ is independent, where $p$, $q$, and $r$ are distinct atomic formulas.

(b) In Second Order Logic, let $s$ be a symbol for a unary function and 0 be a symbol for an individual. The set $\Sigma_{P e a}=\{($ Zer), (Inj),(Ind) $\}$ is independent [Peano 1891]:

$$
\begin{aligned}
\text { (Zer) } & \forall x(\neg s x=0), \\
\text { (Inj) } & \forall x \forall y(s x=s y \rightarrow x=y), \\
\text { (Ind) } & \forall X\{[X 0 \wedge \forall x(X x \rightarrow X s x)] \rightarrow \forall x X x\} .
\end{aligned}
$$

The notion of independence has played an important role in mathematics. We are not aware of any work where the history of this notion has been developed in detail, but whatever this text may be, it cannot fail to mention the examples of non-Euclidean geometries and the non-Cantorian set theories, which emerged from problems of independence of sets of postulates and have profoundly changed the way we think mathematics.

\subsection{Complete Independence (Moore, 1910)}

E.H. Moore generalized Peano's definition in the following manner.

Definition 2 [Moore 1910] $\Sigma$ is completely independent if $\operatorname{Mod}[(\Sigma \backslash X) \cup\{\neg \varphi: \varphi \in$ $X\}] \neq \emptyset$, for every $X \subseteq \Sigma$. 
By restricting $X$ to singletons, we have the same notion of independence from Section 3.1. This justifies the following trivial result.

Theorem 1 If $\Sigma$ is completely independent, then $\Sigma$ is independent.

Example 2 (a) In propositional logic, the independent set $\{p, q,(p \vee q) \wedge r\}$, from Example 1.(a), is not completely independent. In fact, taking $X=\{p, q\}$, the set $\{\neg \boldsymbol{p}, \neg \boldsymbol{q},(p \vee q) \wedge r\}$ is not satisfiable. Given a positive integer $n$, a trivial example of a completely independent set is $\Sigma_{n}=\left\{p_{1}, p_{2}, \ldots, p_{n}\right\}$, where $p_{1}, p_{2}, \ldots, p_{n}$ are distinct atomic formulas.

(b) In First Order Logic, let $R$ be a symbol for a binary relation. The set $\Sigma_{E q u}=$ $\{(\operatorname{Ref}),(\mathrm{Sym}),(\mathrm{Tra})\}$, axiomatizing equivalence relations, is completely independent, where

$$
\begin{aligned}
\text { (Ref) } & \forall x(x R x), \\
\text { (Sym) } & \forall x \forall y(x R y \rightarrow y R x), \\
\text { (Tra) } & \forall x \forall y \forall z((x R y \wedge y R z) \rightarrow x R z) .
\end{aligned}
$$

(c) In Second Order Logic, the independent set $\Sigma_{P e a}$ from Example 1 (b) is not completely independent. Indeed, the set $\{\neg$ (Zer), $\neg($ Inj), (Ind) $\}$ does not have a model (cf. [Cerioli et al. 2021]). But, the set $\Sigma_{\text {Bin }}=\{($ Zer), (Inj), (Bind) $\}$, proposed in [Cerioli et al. 2021], is a completely independent alternative axiomatization, where (Zer) and (Inj) are as in example 1(b) and (Bind) is

$$
\forall X\{[X 0 \wedge \forall x(X x \leftrightarrow X s x)] \rightarrow \forall x X x\} .
$$

\subsection{Primality}

In an abstract presented in a meeting of the American Mathematical Society, H. M. Sheffer proposed a notion of independence based on a concept of mutual primality between postulates:

Two postulates, neither of which implies any part of the other, may be called mutually prime. [Sheffer 1916]

Sheffer based his work on an idea of a postulate implying a part of another. However, he was not very precise as to what he means by this later notion.

We propose an interpretation of primality between sentences throughout an analogy between divisibility and logical implication. Recall that two natural numbers $a$ and $b$ are mutually prime if, for every natural number $c$, if $c \mid a$ and $c \mid b$, then $c=1$. To adapt this notion to senteces, we consider that:

1. A part of a sentence is a non-valid sentence that is a logical consequence of it.

2. Two sentences are mutually prime if they do not have any parts in common.

Based on this interpretation, we propose the following definition:

Definition 3 Two sentences $\varphi$ and $\psi$ are mutually prime if, for every sentence $\theta$, if $\varphi=\theta$ and $\psi \models \theta$, then $\models \theta$.

Lemma 1 Two sentences $\varphi$ and $\psi$ are mutually prime if, and only if, $\models \varphi \vee \psi$.

Proof. Suppose that $\varphi$ and $\psi$ are mutually prime. Since $\varphi=\varphi \vee \psi$ and $\psi \models \varphi \vee \psi$, we have $=\varphi \vee \psi$. Conversely, suppose $=\varphi \vee \psi$. Let $\theta$ be a sentence such that $\varphi \models \theta$ and $\psi \models \theta$. Then, $\varphi \vee \psi \models \theta$. Now, since $\models \varphi \vee \psi$, it follows that $\models \theta$. Therefore, $\varphi$ and $\psi$ are mutually prime. 
Definition 4 A set of postulates $\Sigma$ is prime if it is independent (cf. Section 3.1) and its sentences are pairwise mutually prime.

Example 3 (a) In Propositional Logic, by Lemma 1, none of the sets presented in Examples 1(a) and 2(a), are prime. But, the set $\Sigma_{p r i}=\{p, p \rightarrow q,(p \wedge q) \rightarrow r\}$ is prime.

(b) In First Order Logic (cf. Example 2(b)) it is immediate that $\Sigma_{E q u}$ is not prime.

(c) In Second Order Logic (cf. Examples 1(b) and 2(c)), none of the sets $\Sigma_{P e a}$ and $\Sigma_{B i n}$ are prime. But, the equivalent set $\Sigma_{N a t}=\{(\mathrm{Clos})$, (Dind) $\}$ is prime, where

$$
\begin{array}{ll}
\text { (Clos) } & \exists X[\exists x(X x) \wedge \exists y(\neg X y) \wedge \forall x(X x \rightarrow X s x)] \\
\text { (Dind) } & \forall X[\exists x(X x) \wedge \exists y(\neg X y) \rightarrow \exists x\langle X x \\
& \wedge\{(\neg X s x) \vee[x \neq 0 \wedge \forall y(s y=x \rightarrow \neg X y)]\}\rangle]
\end{array}
$$

In our view, the meaning of "a sentence implying a part of another", in [Sheffer 1916], is too vague for the text to contain a proper definition of mutual primality, so we propose Definition 3. We presume that our concept of primality is different from Sheffer's because, according to [Sheffer 1916], every prime set of postulates should be completely independent, but under Definition 3, primality and complete independence are incompatible (cf. Section 4 ).

\subsection{Irredundance (Church, 1925)}

The next notion of independence is due to A. Church. It extends Peano's independence to a direction opposite to Moore's complete independence. Adopting a somewhat negative semantical point of view, we can say that each postulate in a set $\Sigma$ plays the role of excluding certain structures from being models of $\Sigma$ (a sentence $\varphi \in \Sigma$ excludes a model $\mathfrak{M}$ if $\mathfrak{M} \forall \forall \varphi$ ). But, just as it can be seen as redundant to adopt an axiom that is already implied by previously adopted axioms, it can also be seen as redundant to have a structure be excluded by more than one of the adopted axioms. For a set to be considered irredundant, no structure should be excluded by more than one sentence of the set. In contrast with complete independence (as becomes clear after this section), given any non-trivial $X \subseteq \Sigma$, if $\Sigma$ is completely independent, then $\operatorname{Mod}(\{\neg \varphi: \varphi \in X\}) \neq \emptyset$, and if $\Sigma$ is irredundant, then $\operatorname{Mod}(\{\neg \varphi: \varphi \in X\})=\emptyset$. Irredundance aims to have different postulates excluding disjoint classes of structures. The more precise and slightly indirect definition of irredundance given by Church requires a couple of preliminary concepts.

Definition 5 [Church 1925] A sentence $\psi$ is weaker than a sentence $\varphi$ if $\varphi \models \psi$ and $\psi \not \models \varphi$.

Definition 6 [Church 1925] A sentence $\varphi$ can be weakened with respect to a set $\Sigma$ if there exists a sentence $\psi$ such that: (1) $\psi$ is weaker than $\varphi$, and (2) $(\Sigma \backslash\{\varphi\}) \cup\{\psi\} \models \varphi$.

Definition 7 [Church 1925] A set of sentences $\Sigma$ is irredundant if: (1) $\Sigma$ is independent (cf. Section 3.1) and (2) $\varphi$ cannot be weakened with respect to $\Sigma$, for any $\varphi \in \Sigma$.

Church proved, in [Church 1925], the following theorem restricted to the case where $\Sigma$ is a finite set. Nevertheless, we proved that the theorem holds regardless of the cardinality of $\Sigma$.

Theorem 2 Let $\Sigma$ be a set of sentences. A sentence $\varphi$ in $\Sigma$ cannot be weakened with respect to $\Sigma$ if and only if for every $\psi$ in $\Sigma \backslash\{\varphi\}$, it holds that $\neg \varphi \models \psi$.

Corollary 1 A set of sentences is irredundant if and only if it is prime. 


\subsection{Strong and absolute independence (Harary, 1961)}

The two last notions of independence we examine were defined by $F$. Harary [Harary 1961]. In a certain sense, these are only partial notions, because they are restricted to postulates having the form "if $\varphi$, then $\psi$ " ( $\varphi$ is allowed to be a logically valid sentence).

Strong independence aims to be a stronger form of Peano's notion of independence. The key difference being the use of a special kind of negation-which we call strong negation - that captures Harary's concept of an entailment that never holds:

We shall say that an axiom $A$ of the form " $p$ implies $q$ " never holds in a model $M$ if the hypothesis $p$ occurs at least once in $M$ and furthermore whenever $p$ is true, $q$ is false, i.e., $p$ implies not $q$. [Harary 1961]

Absolute independence is, then, defined analogously to complete independence (cf. Section 3.2), the only difference being the use of the strong negation in place of the classical negation.

Definition 8 A sentence $\varphi$ has a strong negation if there exist a natural number $n$ and two formulas $A$ and $B$ such that all free variables occurring in them are among the variables $x_{1}, \ldots, x_{n}$ and $\varphi$ is $\forall x_{1} \ldots \forall x_{n}(A \rightarrow B)$. In this case, the strong negation of $\varphi$, denoted by $\sim \varphi$, is $\exists x_{1} \ldots \exists x_{n} A \wedge \forall x_{1} \ldots \forall x_{n}(A \rightarrow \neg B)$.

Definition 9 [Harary 1961] A satisfiable set of postulates $\Sigma$, such that every postulate in $\Sigma$ has a strong negation, is strongly independent (or very independent) if, $\Sigma \backslash\{\varphi\} \cup\{\sim \varphi\}$ is satisfiable, for every $\varphi$ in $\Sigma$.

Definition 10 [Harary 1961] A satisfiable set of postulates $\Sigma$, such that every postulate in $\Sigma$ has a strong negation, is absolutely independent if, the set $(\Sigma \backslash X) \cup\{\sim \varphi: \varphi \in X\}$ is satisfiable, for every subset $X \subseteq \Sigma$.

Example 4 (a) In [Harary 1961], Harary argues that the set of axioms $\Sigma_{E q u}$, for equivalence relations (cf. Example 2(b)), is not strongly independent. Indeed, the set $\{(\mathrm{Ref}), \sim(\mathrm{Sym}),(\mathrm{Tra})\}$ does not have a model because, as in any reflexive relation $x R x$ holds for every element $x$, then $x R y \rightarrow \neg y R x$ would not hold when the elements $x$ and $y$ coincide. So, Harary adapted the axioms defining the notion of an equivalence relation, in order to produce an absolutely independent set. In First Order Logic (cf. Example 2(b)) consider the set $\Sigma_{E q u}^{\mathrm{H}}=\{(\operatorname{RefH}),(\mathrm{SymH}),(\mathrm{TraH})\}$, where

$$
\begin{aligned}
(\text { RefH }) & \forall x(x=x \rightarrow x R x), \\
(\text { SymH }) & \forall x \forall y[(x \neq y \wedge x R y) \rightarrow y R x], \\
(\text { TraH }) & \forall x \forall y \forall z[(x \neq y \wedge x \neq z \wedge y \neq z \wedge x R y \wedge y R z) \rightarrow x R z] .
\end{aligned}
$$

The set $\Sigma_{E q u}^{\mathrm{H}}$ is absolutely independent.

(b) The sets $\Sigma_{P e a}$ and $\Sigma_{B i n}$ are not strongly independent.

\section{Comparing the definitions}

In this section, we compare, from the point of view of logical strength, the notions of independence presented in Section 3 . 
By Theorem 1, and Definitions 4 and 7, Peano's notion of independence is weaker than Moore's complete independence, the notion of primality, and Church's notion of irredundance. In Corollary 1 , we proved that the notions of irredundance and primality are equivalent. Harary's definitions of independence can be easily compared to those of Peano and Moore. Notice that, for every sentence $\varphi$, its strong negation $\sim \varphi$ entails its classical negation $\neg \varphi$, which guarantees both that strong independence implies independence, and absolute independence implies complete independence.

As mentioned in [Church 1925], the notions of complete independence and irredundance are incompatible. We present a proof based on our notion of primality.

Theorem 3 Let $\Sigma$ be a set of sentences with at least two postulates. If $\Sigma$ is prime, then it is not completely independent.

Proof. Suppose that $\Sigma$ is prime. Let $\varphi, \psi \in \Sigma$ be distinct sentences. Then, $\models \varphi \vee \psi$ and therefore, $\operatorname{Mod}((\Sigma \backslash\{\varphi, \psi\}) \cup\{\neg \varphi, \neg \psi\})=\emptyset$.

Complete independence seems to match more accurately the intuitive literal meaning of "independence." It reflects the idea of facts or objects having no connection or influence over one another. Whereas, intuitively, primality (or irredundance) addresses the possible overlaps between sentences, when sentences are seen as descriptions of their own models. More precisely, primality addresses the overlaps between the classes of models prohibited by each of the axioms in consideration.

A perspective of these results can be seen in the diagram below. All of the numbered regions represented in the diagram have been found to be non-empty.

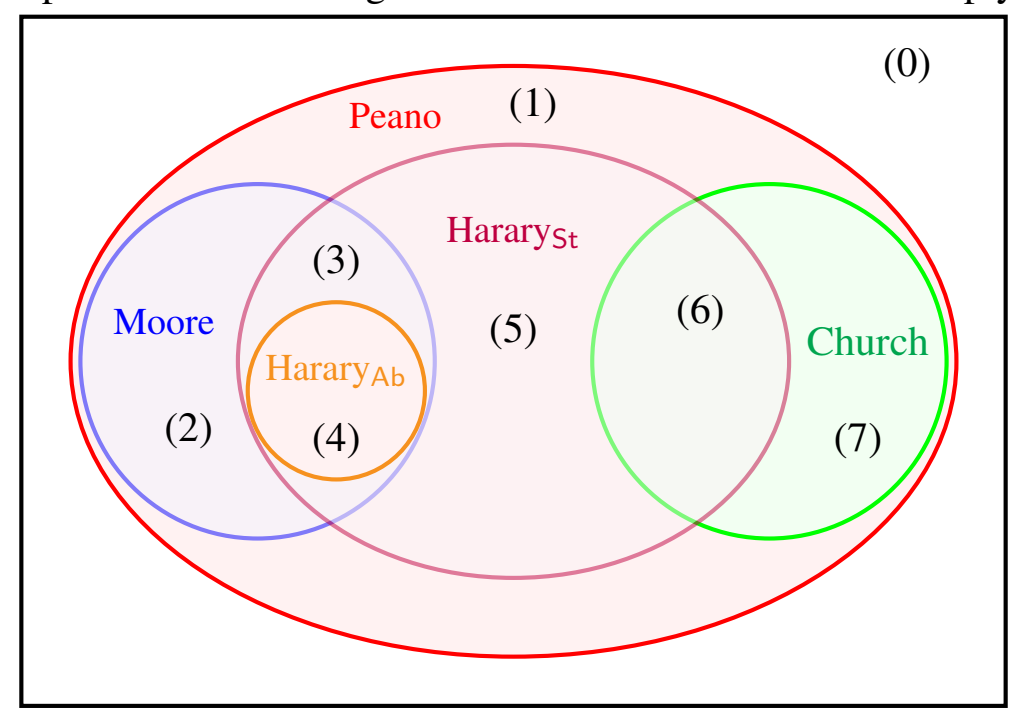

In this diagram, each colored region corresponds to one of the definitions of independence. Since Harary has two notions of independence, there are two regions named after him: for strong independence we have the region labelled Hararyst; and for absolute independence we have the region labelled Harary ${ }_{A b}$. The topological relations between the colored regions summarize the implications or incompatibilities mentioned above. For instance, the region assigned to Harary's absolute independence is contained in the intersection between the regions of Moore's complete independence and Harary's strong independence, because every absolutely independent set is both completely and strongly independent. The regions of complete independence and irredundance do not overlap because by Theorem 3 , non-trivial completely independent sets are not irredundant. 


\section{Exploring the landscape}

\subsection{On irredundance}

Harary remarks in [Harary 1961] that, even though the usual set of axioms for the theory of equivalence relations is completely independent (cf. Example 2(b)), the axioms of symmetry and transitivity together imply that reflexivity holds at least partially. More precisely, every element that is related to some other by a symmetric and transitive relation must also be related to itself. This partial implication is reminiscent of Sheffer's description of mutual primality:

Two postulates, neither of which implies any part of the other, may be called mutually prime. [Sheffer 1916]

In this example we have the sentence $\forall x[(\exists y x R y) \rightarrow x R x]$ that is both a consequence of the set $\{(\operatorname{Ref})\}$ and of the set $\{(\mathrm{Sym}),(\mathrm{Tra})\}$. The redundance shown here lies in the fact that $\{(\operatorname{Ref}),(\mathrm{Sym}),(\mathrm{Tra})\}$ has two distinct subsets that are minimal among those that imply $\forall x[(\exists y(x R y)) \rightarrow x R x]$.

With the following theorem, we show that this would not happen to an irredundant set of axioms. In fact, the irredundance (or primality) of a set of axioms is a necessary and sufficient condition for the existence and uniqueness of a minimal subset of the axioms that imply a given sentence, namely the intersection of all such subsets.

Theorem 4 An independent set of axioms $\Sigma$ is irredundant if and only if, for every sentence $\varphi$ such that $\Sigma \mid=\varphi$, the set $\Gamma=\{\psi \in \Sigma \mid \not \forall \varphi \vee \psi\}$ is the smallest subset of $\Sigma$ such that $\Gamma \models \varphi$ (i.e., if $\Delta \models \varphi$, then $\Gamma \subseteq \Delta$, for all $\Delta \subseteq \Sigma$ ).

ProOF. Let $\Sigma$ be an independent set of sentences. Suppose that $\Sigma$ is irredundant. Let $\varphi$ be a sentence such that $\Sigma \mid=\varphi$ and let $\Gamma=\{\psi \in \Sigma|\not| \varphi \vee \psi\}$. Let $\mathfrak{M} \in \operatorname{Mod}(\Gamma)$. If $\mathfrak{M} \models \Sigma$, then $\mathfrak{M} \models \varphi$. And, if $\mathfrak{M} \not \models \Sigma$, then $\mathfrak{M} \forall \psi$ for some $\psi \in \Sigma \backslash \Gamma$, and since $\models \varphi \vee \psi$ (because $\psi \notin \Gamma$ ), we have $\mathfrak{M} \models \varphi$. Thus, $\Gamma \models \varphi$. Let $\Delta \subseteq \Sigma$ be such that $\Delta \models \varphi$. Suppose, for a contradiction, that there exists a sentence $\psi \in \Gamma \backslash \Delta$. Then, since $\psi \in \Gamma$, there is a structure $\mathfrak{M}$ such that $\mathfrak{M} \not \forall \varphi \vee \psi$. Since $\Sigma$ is irredundant, for every $\theta \in \Sigma \backslash\{\psi\}$ it holds that $=\psi \vee \theta$. And since $\Delta \subseteq \Sigma \backslash\{\psi\}$, then $\neg \psi \models \Delta$. Then $\mathfrak{M} \models \Delta$. But since $\Delta \models \varphi$, then $\mathfrak{M} \models \varphi$, which contradicts the hypothesis that $\mathfrak{M} \forall \varphi \vee \psi$. Therefore, $\Gamma \subseteq \Delta$.

Conversely, suppose that, for every sentence $\varphi$, the set $\Gamma=\{\psi \in \Sigma \mid \not \forall \varphi \vee \psi\}$ is the smallest subset of $\Sigma$ such that $\Gamma \models \varphi$. Since $\Sigma$ is independent, it suffices to show that all pairs of distinct sentences in $\Sigma$ are mutually prime in order to prove that $\Sigma$ is irredundant. Let $\varphi, \psi \in \Sigma$ be two distinct sentences. Since $\{\varphi\} \models \varphi \vee \psi$ and $\{\psi\} \models \varphi \vee \psi$, it follows from the hypothesis that $\Gamma_{\varphi \vee \psi} \subseteq\{\varphi\} \cap\{\psi\}=\emptyset$, and $\Gamma_{\varphi \vee \psi}=\varphi \vee \psi$, where $\Gamma_{\varphi \vee \psi}=\{\theta \in \Sigma \mid \not \models(\varphi \vee \psi) \vee \theta\}$. So $\varphi \vee \psi$ is valid, and therefore $\varphi$ and $\psi$ are mutually prime. We conclude that $\Sigma$ is irredundant.

\subsection{On strong independence}

The motivation given for Theorem 4 in Section 5.1 indicates some similarities between the possible intentions attributed to irredundance and strong independence. In the following theorem, we show that, under some mild conditions, irredundance implies strong independence. 
Theorem 5 Let $\Sigma$ be a set of sentences such that every sentence in $\Sigma$ has a satisfiable strong negation. If $\Sigma$ is irredundant, then $\Sigma$ is strongly independent.

Proof. Suppose that $\Sigma$ is irredundant. Let $\varphi \in \Sigma$. Assume, for a contradiction, that $\operatorname{Mod}((\Sigma \backslash\{\varphi\}) \cup\{\sim \varphi\})=\emptyset$. This means that $\Sigma \backslash\{\varphi\} \models \neg \sim \varphi$. Since $\sim \varphi \models \neg \varphi$, we also have $\{\varphi\} \models \neg \sim \varphi$. By Theorem 4, there is a set $\Gamma \subseteq \Sigma$ such that $\Gamma \subseteq(\Sigma \backslash\{\varphi\}) \cap\{\varphi\}=\emptyset$ and $\Gamma \models \neg \sim \varphi$. Thus, $\models \neg \sim \varphi$, contradicting the hypothesis that $\sim \varphi$ is satisfiable. We conclude that $\Sigma$ is strongly independent.

The converse of Theorem 5 does not hold. As a counter-example, we could use any absolutely independent set, such as $\Sigma_{E q u}^{\mathrm{H}}$ in Example 4 (a). As we have mentioned in Section 4, absolutely independent sets are both strongly independent and completely independent. And since non-trivial completely independent sets cannot be irredundant (cf. Theorem 3), then any non-trivial absolutely independent set is an example of a strongly independent set that is not irredundant.

Harary's definitions are very sensitive to syntax. Both definitions of strong independence and absolute independence rely on the notion of a sentence "never holding" in a model, which is not invariant under semantical equivalence. This brings up some unwanted consequences for the notions of strong and absolute independence. As an example of this, consider Harary's axiom for 'distinctly transitive' relations:

$$
\text { (TraH) : } \forall x \forall y \forall z((x \neq y \wedge y \neq z \wedge x \neq z \wedge x R y \wedge y R z) \rightarrow x R z) .
$$

This sentence, as any other, is semantically equivalent to its contrapositive:

$$
\psi: \forall x \forall y \forall z(\neg x R z \rightarrow(x=y \vee y=z \vee x=z \vee \neg x R y \vee \neg y R z)) .
$$

As we have shown in example 4 of Section 3.5, there are models in which $(\mathrm{TraH})$ never holds, that is, the strong negation of (TraH) holds, but the strong negation of $\psi$ is not even satisfiable:

$$
\sim \psi: \exists x \exists y \exists z(\neg x R z) \wedge \forall x \forall y \forall z(\neg x R z \rightarrow(x \neq y \wedge y \neq z \wedge x \neq z \wedge x R y \wedge y R z)) .
$$

Even though $\{(\mathrm{RefH}),(\mathrm{SymH}),(\mathrm{TraH})\}$ is absolutely independent and $(\mathrm{TraH})$ is semantically equivalent to $\psi$, the set $\{(\operatorname{RefH}),(\mathrm{SymH}), \psi\}$ is not even strongly independent.

\section{References}

Cerioli, M. R., Nobrega, H., Silveira, G., and Viana, P. (2021). On the (in)dependence of the peano axioms for natural numbers. Accepted for publication in The History and Philosophy of Logic.

Church, A. (1925). On irredundant sets of postulates. Transactions of the American Mathematical Society, 27(3):318-328.

Harary, F. (1961). A very independent axiom system. The American Mathematical Monthly, 68(2):159-162.

Moore, E. H. (1910). Introduction to a form of general analysis, volume 2. Yale University Press.

Peano, G. (1889). Arithmetices principia: Nova methodo exposita. Fratres Bocca.

Peano, G. (1891). Sul concetto di numero. Rivista Matematica, 1:256-267.

Sheffer, H. M. (1916). Mutually prime postulates. Bulletin of the American Mathematical Society, 22:287. 